\title{
Research on the operational regional coverage of satellite and spacecraft tracking and controlling
}

\author{
Lihua $\mathrm{Qi}^{1{ }^{1,}}$ Dongqiu Xing ${ }^{1}$, Rui Wang ${ }^{2}$ and Jingna Cui ${ }^{1}$ \\ ${ }^{1}$ Information Communications Academy, National University of Defense Technology, No.5 \\ Guangming Road, Wangqu Town, Chang'an District, Xi'an City, 710106, China \\ ${ }^{2}$ College of Optoelectronic Engineering, Chengdu University of Information Technology, No.24 \\ Block 1,Xuefu Road, Chengdu City, 610225, China
}

Keywords: Ground station, Flare angle, Coverage, Measurement and control circles.

\begin{abstract}
In view of the problem of building ground stations for tracking and controlling of satellites and spacecraft, considering the fixed angle between the orbit of the satellite or spacecraft and the equatorial surface of the earth, and the difference of longitude between the two circles in succession of the satellite or spacecraft caused by the rotation of the earth, the operation area of the satellite or spacecraft was calculated by using the method of spherical projection of satellite orbit rotation, taking the earth as the reference system. The minimum number of ground stations needed for satellite tracking and controlling was calculated in three cases, by using the mathematical model of sphere ring area and honeycomb coverage. This model was validated by the launch and operation data of Shenzhou 7.
\end{abstract}

\section{Introduction}

With the development of the aerospace industry, the application of satellites and spacecraft plays an irreplaceable role in many fields. Launching and operation of satellites and spacecraft was a complex system engineering, and measurement and control was an important part of the whole system. In this paper, the whole process measurement and control of satellites or spacecraft using fewer ground stations was studied. The ideal situation was to track and control satellites and spacecraft (especially manned spacecraft) in the whole process. However, the measurement and control equipment could only observe the airspace above the tangent plane of the point, and the measurement and control effect was not good within the range of 3 degrees included angle with the ground plane. In fact, the measurement and control range of each measurement and control station only considered the airspace with an angle of more than 3 degrees with the ground plane. During the launch and operation of satellites or spacecraft, there were often multiple ground stations jointly completing measurement and

\footnotetext{
*Corresponding author: lihuaqizhf@,126.com
} 
contral tasks, In reference [1], the ground stations when the orbit of the ground station was coplanar with that of the satellite or spacecraft, and the orbit was circular, were discussed. In references [2] and [3], the ground stations were discussed the when the orbit was ellipse. In reference [4], the influence of earth rotation was considered, but there was a blind area in the measurement and control area of the station. Therefore, considering the influence of the earth's rotation, how many ground stations were needed to establish to achieve no blind area measurement and control?

\section{Analysis of the operation orbit of a satellite or spacecraft}

Generally, the orbit of satellite or spacecraft had a fixed angle with the equatorial plane of the earth, and it operates on a spheres $\mathrm{S}$ with a height of $\mathrm{h}$ above the ground. There would be some differences in the longitude of two successive cycles of a satellite or spacecraft, because of the rotation of the earth, Therefore, in the case of taking the earth as a reference, it was reflected that the orbit of the satellite rotates around the earth axis, and after several cycles, the orbit of the satellite was on a spherical surface, as shown in Fig. 1., where $\beta$ was the angle between the orbit of a satellite or spacecraft and the equatorial plane of the earth. The measurement and control range of the ground measurement and control station only considered the airspace with an angle of more than 3 degrees with the ground plane, the measurement and control range of the station was a cone with the measurement and control station as the vertex and the included angle of the generatrix as $\theta$. The spherica crown was obtained by intersecting the operation spherical surface of the satellite and the cone bottom. Set the diameter of the bottom surface of the spherical crown as $l_{2}$ and the bottom surface of the spherical crown as a circular area, that is, the measurement and control circle, as shown in Fig. 2.,

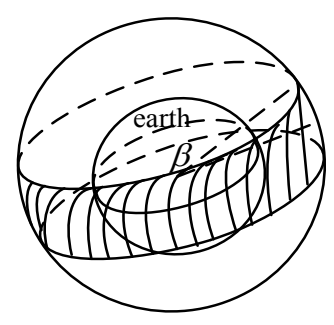

Fig. 1. The orbit of a satellite or spacecraft.

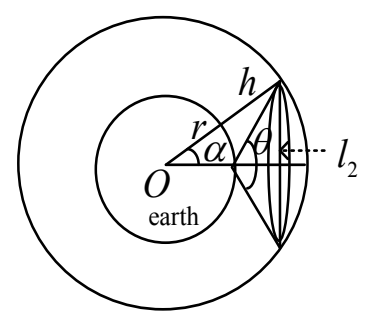

Fig. 2. Measurement range of single ground station.

According to the relationship between triangles in Fig. 2.,

$$
l_{2}=2(r+h) \sin \alpha
$$


When $\theta=174^{\circ}$, i.e $\theta=0.97 \pi$, the number of ground stations was the least,

$$
\begin{gathered}
\alpha=\frac{\theta}{2}-\arcsin \left(\frac{r \cdot \sin \left(\frac{\theta}{2}\right)}{r+h}\right)=0.485 \pi-\arcsin \left(\frac{r \sin (0.485 \pi)}{r+h}\right) \\
l_{2}=2(r+h) \sin \alpha=2(r+h) \sin \left[0.485 \pi-\arcsin \left(\frac{r \sin (0.485 \pi)}{r+h}\right)\right]
\end{gathered}
$$

so the measurement and control range of each ground station was the same.

Considering seamless coverage, the method was as follows: The circular track surface of the satellite or spacecraft was expanded according to the circumference of both ends, and it was a curved surface, then we projected the expanded surface onto the ground to get a rectangular region, where the length of the rectangular region was the perimeter of both ends of the spherical ring region, set as $b$, and the width was the chord length corresponding to the spherical ring region, set as $\alpha$, as shown in Fig. 3..

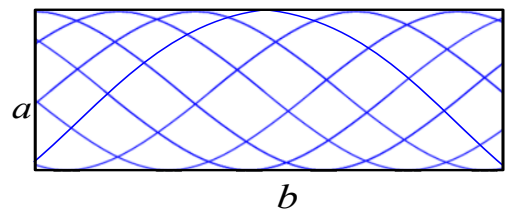

Fig. 3. Projection plan of satellite operation area.

Combined with Figure 1 and Figure 2

$$
a=2(r+h) \sin \beta, \quad b=2 \pi(r+h) \cos \beta
$$

According to the characteristics of the sphere ring region, to cover the whole sphere ring region, only the projection rectangle region of the sphere region needed to be covered.

\section{Solution to the number of ground stations}

If the ground station covered the low track area, it would be able to cover the high track area .So the rectangular area of the projection of the expanded spherical ring surface was covered, the spherical ring surface also could be covered. Using this principle, when the field angle of the ground station was the largest, that is $\theta=0.97 \pi$, the corresponding chord length was compared with the width of the rectangular region, then the problem was transformed into the problem of covering the rectangular area with the least measurement and control circles. The number of circles was the number of the ground station.

\subsection{The two sides of the rectangular domain and the measurement and control circle were tangent}

(1) The two sides of the rectangular domain and the measurement and control circle were tangent, that was $l_{2}=a$. In order to fully cover the rectangular domain, the order of the measurement and control circle should be as shown in Fig. 4., 


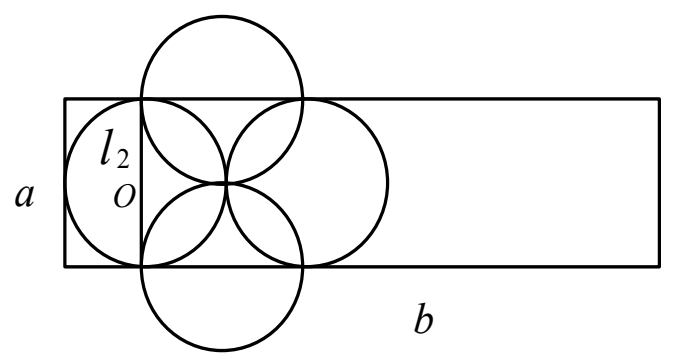

Fig. 4. $l_{2}=a$, arrangement chart of measurement and control circle.

From $l_{2}=a, 2(r+h) \sin \alpha=2(r+h) \sin \beta, \sin \alpha=\sin \beta$,

$$
\beta=\alpha=0.485 \pi-\arcsin \left(\frac{r \sin (0.485 \pi)}{r+h}\right)
$$

according to the order of measurement and control circles, the number of measurement and control circles covering rectangular area was obtained, that was, the number of ground stations as follow,

$$
n=\frac{6 \pi(r+h) \cos \beta}{2(r+h) \sin \alpha}=3 \pi \cot \beta=3 \pi \cot \left[0.483 \pi-\arcsin \left(\frac{r \sin 0.485 \pi}{r+h}\right)\right]
$$

when $n$ was an integer, $n=n$; when $n$ was a non integer, $n=[n]+1$.

\subsection{The two sides of the measurement and control circle and the rectangular domain intersect}

When the two sides of the measurement and control circle and the rectangular domain intersect, that was $l_{2}>a$, the order of the measurement and control circle should be as shown in Fig. 5., in order to fully cover the rectangular domain.

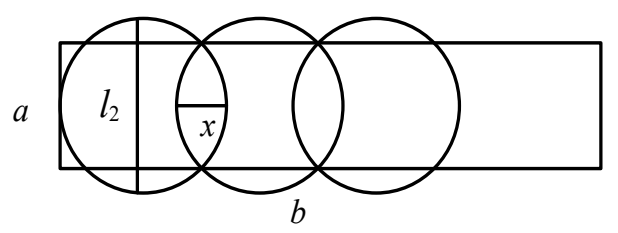

Fig. 5. $l_{2}>a$, arrangement chart of measurement and control circle.

From $l_{2}=2(r+h) \sin \alpha, x=2\left(\frac{l_{2}}{2}-\sqrt{\left(\frac{l_{2}}{2}\right)^{2}-\left(\frac{a}{2}\right)^{2}}\right)$,so,

$$
n=\frac{b}{l_{2}-x}=\frac{2 \pi \cos \beta(r+h)}{2 \sin \alpha(r+h)-2\left(\frac{l_{2}}{2}-\sqrt{\left(\frac{l_{2}}{2}\right)^{2}-\left(\frac{a}{2}\right)^{2}}\right)}
$$


when $n$ was an integer, $n=n$; when $n$ was a non integer, $n=[n]+1$.

\subsection{Arrangement method of regular hexagon of the measurement and control circle}

When $l_{2}<\alpha$, in order to fully cover the rectangular area, the inscribed regular hexagon of the measurement and control circle was connected arrangement method to cover the rectangular area[5], that was honeycomb cover, and there was no gap or overlap between the regular hexagons, very suitable for regional planning and grid division, as long as the measurement and control radius of the measurement and control station was determined, the minimum number of measurement and control station could be used to monitor the target area. Such grid planning did not affect the circular monitoring coverage area of the measurement and control station, the simplified regular hexagon was just to make the planning graphics more intuitive, The arrange order of regular hexagon should be as shown in Figure 6, the number of ground stations wascalculated in four cases, a was divided by $\frac{3}{2} \cdot \frac{l_{2}}{2}$, b was divided by $\sqrt{3} \cdot \frac{l_{2}}{2}$.
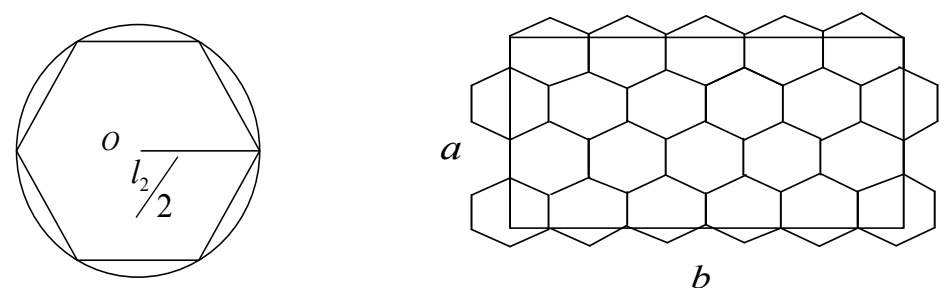

Fig. 6. Array order of regular hexagon.

Situation 1: $a$ and $b$ could be divided by one unit respectively;

$$
\left\{\begin{array}{l}
n_{1}=\frac{b}{\sqrt{3} \cdot \frac{l_{2}}{2}}, \quad m_{1}=\frac{a}{\frac{3}{2} \cdot \frac{l_{2}}{2}} \\
n=n_{1} \times m_{1}
\end{array}\right.
$$

Situation 2: $a$ could be divided by one unit, but $b$ couldn't;

$$
\left\{\begin{array}{l}
n_{1}=\frac{b}{\sqrt{3} \cdot \frac{l_{2}}{2}}, \quad m_{1}=\frac{a}{\frac{3}{2} \cdot \frac{l_{2}}{2}} \\
n=\left(\left[n_{1}\right]+1\right) \times m_{2}
\end{array}\right.
$$

Situation 3: $b$ could be divided by one unit, but $a$ couldn't;

$$
\left\{\begin{array}{l}
n_{1}=\frac{b}{\sqrt{3} \cdot \frac{l_{2}}{2}}, m_{1}=\frac{a}{\frac{3}{2} \cdot \frac{l_{2}}{2}} \\
n=n_{1} \times\left(\left[m_{1}\right]+1\right)
\end{array}\right.
$$


Situation 4: both $a$ and $b$ couldn't be divided by one unit respectively.

$$
\left\{\begin{array}{l}
n_{1}=\frac{b}{\sqrt{3} \cdot \frac{l_{2}}{2}}, \quad m_{1}=\frac{a}{\frac{3}{2} \cdot \frac{l_{2}}{2}} \\
n=\left(\left[n_{1}\right]+1\right) \times\left(\left[m_{1}\right]+1\right)
\end{array}\right.
$$

In summary, When there was a fixed angle between the orbit of satellites or spacecraft and the equatorial plane of the earth, According to the relationship between the width a of the projection area of the orbit of satellite or spacecraft and $l_{2}$, the value of $\mathrm{n}$ was calculated by takeing $a$ and $l_{2}$ into the corresponding expressions, this was the number of ground station.

According to the modle of the front, the operation data of Shenzhou 7 was collected [6], the height of Shenzhou 7's orbit was about 343 kilometers from the earth's surface, The angle between the orbit plane and the equatorial plane of the earth was $42.2^{\circ}$, the value of $a, b$, $l_{2}$ was calculated by bring in data, so $a=9019 \mathrm{~km}, b=31251 \mathrm{~km}, l_{2}=3551 \mathrm{~km}$, because $a>l_{2}$ using situation 4 .

$$
n=\left\{\left[\frac{2 b}{\sqrt{3} l_{2}}\right]+1\right\} \times\left\{\left[\frac{4 a}{3 l_{2}}\right]+1\right\}=44
$$

At least 44 ground stations were needed to be set up for tracking and controlling Shenzhou 7.

\section{Summary}

For the tracking and controlling of satellites or spacecraft, we had established model to achieve no blind area measurement and control,considering the fixed angle between the orbit of the satellite or spacecraft and the equatorial surface of the earth, and the difference of longitude between the two circles in succession of the satellite or spacecraft caused by the rotation of the earth, the operation area of the satellite or spacecraft was calculated by using the method of spherical projection of satellite orbit rotation, taking the earth as the reference system. On this basis, the relationship between the width of the projection plane of the operation area and the chord length of the satellite orbit corresponding to the maximum opening angle of the ground station was analyzed, the minimum number of ground stations was calculated In three cases, when the satellite orbit height was h,The angle between the orbit plane and the equatorial plane of the earth was $\beta$, the functional relationship between the satellite orbit height and the minimum number of ground stations was obtained.

\section{References}

1. WANG Yu-mei, "Tracking and Telemetering of Satellites and Spacecraft," Journal of Hubei University for Nationalities, vol.26, Issue 1, pp.67-69, 2011.

2. WANG Juan. "Analysis of Tracking and Monitoring Satellites and Spaceships," Journal of An qing Teachers College, vol.18, Issue 1, pp.59-63, 2012. 
3. ZHU Jian-guo. JIN Yue-qiang. "The Mathematical Model on the Distribution of Satellite Monitoring Station," Journal of Nanjing Institute of Industry Technology, vol.10, Issue 2, pp.64-66, 2010.

4. WANG Xiu-qin. "Tracking and Telemetering of Satellites and Spacecraft," Journal of Chifeng University, vol.28, Issue 2, pp.138-140, 2012.

5. ZHAI Zheng- yi, ZHANG Lun. "A Method of Regular Hexagonal Grid Plot in Wireless Sensor Network," Network and Communication Security, Issue 19, pp.89-107, 2007.

6. The communication scale of ShenZhou 7 was the largest space mission in China, http://www.ck365.cn. 